\title{
OESOPHAGUS
}

\section{Timed barium oesophagram: better predictor of long term success after pneumatic dilation in achalasia than symptom assessment}

\author{
M F Vaezi, M E Baker, E Achkar, J E Richter
}

Gut 2002;50:765-770

See end of article for authors' affiliations

.....................

Correspondence to: Dr M F Vaezi, Department of Gastroenterology, The Cleveland Clinic

Foundation, 9500 Euclid

Ave, Cleveland, $\mathrm{OH}$

44195, USA

Vaezim@CCF.org

Accepted for publication 7 August 2001

\begin{abstract}
Background: Symptom relief post pneumatic dilation is traditionally used to assess treatment success in achalasia patients. Recently, we showed that symptom relief and objective oesophageal emptying are concordant in about $70 \%$ of patients, while up to $30 \%$ of achalasia patients report near complete symptom relief despite poor oesophageal emptying of barium.

Aims: We now report the results of long term clinical follow up in these two groups of achalasia patients, assessing differences in symptomatic remission rates.

Methods: Achalasia patients undergoing pneumatic dilation since 1995 were evaluated both symptomatically and objectively at regular intervals. Pre and post dilation symptoms were recorded. Barium column height was measured five minutes after ingesting a fixed volume of barium per patient to assess oesophageal emptying. Patients who initially reported near complete symptom relief were divided into two groups based on objective findings on barium study: (1) complete oesophageal emptying (concordant group), and (2) poor oesophageal emptying (discordant group). Patients were followed prospectively for symptom recurrence.

Results: Thirty four patients with complete symptom relief post pneumatic dilation were identified. In $22 / 34(65 \%)$ patients, the degree of symptom and barium height improvements was similar (concordant group). In 10/34 (30\%) patients, there was < 50\% improvement in barium height (discordant group). Significantly $(p<0.001)$ more discordant $(9 / 10 ; 90 \%)$ than concordant $(2 / 22 ; 9 \%)$ patients failed therapy at the one year follow up. Seventeen of $22(77 \%)$ concordant patients were still in remission while all discordant patients had failed therapy by six years of follow up. Length of time in symptom remission (mean (SEM)) post pneumatic dilation was significantly $(p=0.001)$ less for the discordant group (18.0 (3.6) months) compared with the concordant group (59.0 (4.8) months).

Conclusions: (1) Poor oesophageal emptying is present in nearly $30 \%$ of achalasia patients reporting complete symptom relief post pneumatic dilation. (2) The majority $(90 \%)$ of these patients will fail within one year of treatment. (3) Timed barium oesophagram is an important tool in the objective evaluation of achalasia patients post pneumatic dilation.
\end{abstract}

A chalasia is a primary oesophageal motor disorder of unknown aetiology characterised by absent oesophageal peristalsis and abnormal lower oesophageal sphincter (LOS) relaxation. ${ }^{12}$ All current treatment options for achalasia are limited to reducing the pressure gradient across the LOS, thus facilitating passive oesophageal emptying by gravity. This goal can be accomplished most effectively by pneumatic dilation and surgical myotomy, or less effectively by pharmacological agents injected endoscopically into the LOS (botulinum toxin) or taken orally (calcium channel blockers and nitrates). ${ }^{23}$

Pneumatic dilation is the most effective non-surgical treatment option for patients with achalasia. Traditionally, patients' reports of symptom improvement have been used to assess the success of pneumatic dilation post therapy. ${ }^{1-5}$ Using this criterion, reports of one year treatment success with the most commonly available pneumatic dilator, Rigiflex balloons (Microvasive, Millford, Massachusetts, USA), vary from $50 \%$ to $93 \%{ }^{2}$ However, recent studies show that many achalasia patients have altered vagal afferent response ${ }^{67}$ resulting in poor reliability of patients' subjective reports. Therefore, objective assessment of oesophageal function may be important in evaluating oesophageal emptying after therapy.

We have previously reported on the technique of using a timed barium swallow as an objective means of assessing oesophageal emptying. ${ }^{8}$ The technique is simple, non-invasive, widely available, and provides an objective measure of success after pneumatic dilation. Using this simple technique in patients one month after pneumatic dilation, we showed that up to $30 \%$ of achalasia patients still had poor oesophageal emptying of barium despite reporting $90-100 \%$ symptom improvement. ${ }^{9}$ We hypothesised that this group would have a relapse of their symptoms earlier when followed long term compared with patients whose symptom resolution correlated with marked improvement in oesophageal emptying.

Therefore, the primary aim of this study was to determine if the timed barium oesophagram is a better predictor of long term treatment success after therapy than symptom assessment alone. In a group of patients with achalasia requiring pneumatic dilation, we correlated patients' reports of symptom improvement with oesophageal barium emptying at one month post therapy. All patients with at least 90-100\% symptom relief were then followed for up to six years to compare the frequency of relapse based on the post procedure oesophagram.

\section{MATERIALS AND METHODS \\ Patient population}

All patients with achalasia who had undergone pneumatic dilation from September 1995 to June 1998 were potential

Abbreviations: LOS, lower oesophageal sphincter 
participants in the study $(n=114)$. Only those with a recent diagnosis of achalasia and no prior therapy qualified for the study $(n=51)$. The diagnostic criteria for achalasia were incomplete LOS relaxation and aperistalsis of the oesophageal body on manometry and the presence of oesophageal dilation, aperistalsis of the oesophageal body, and narrowed LOS on barium oesophagram. Exclusion criteria included unwillingness to participate in the study (two patients), secondary achalasia (one patient), and unavailability of barium study or incomplete symptom questionnaires pre or post dilation (two patients). Therefore, 46 patients constituted the initial study group $^{8}$ of which 32 patients reported near complete symptom resolution post therapy and were followed long term.

All patients completed a standardised pretreatment evaluation consisting of clinical symptom assessment, oesophageal manometry, and timed barium oesophagram. Patients were initially evaluated one month after pneumatic dilation for symptom improvement and underwent an additional timed barium oesophagram. The degree of patient symptom improvement post therapy was recorded and correlated with improvement in oesophageal barium emptying. This was done to assess the utility of timed barium oesophagram in predicting short term clinical remission. Patients reporting near complete $(91-100 \%)$ symptom improvement post pneumatic dilation $(\mathrm{n}=32)$ were then categorised into two groups: (1) those with concordant improvement of oesophageal emptying by barium oesophagram $(n=22)$ and (2) those with poor emptying of oesophageal barium (discordant group) $(n=10)$. These two groups $(n=32)$ constituted the primary study population which were then followed prospectively for symptom recurrence. Repeat intervention was performed only with symptom recurrence. For all discordant and concordant groups of patients having symptom recurrences and in a subgroup $(n=10)$ of concordant patients with continued symptom improvement, timed barium oesophagrams were repeated. This was done to correlate patients' reports of symptom improvement and oesophageal emptying in those followed long term $(n=32)$

\section{Subjective assessment}

All patients were interviewed before treatment and one month after pneumatic dilation. The total symptom scores, consisting of the sum of scores for dysphagia, regurgitation, and chest pain, were recorded. The frequency of each symptom was graded on a scale ranging from 0 to $5(0=$ none; $\mathrm{l}=$ once per month or less; $2=$ once per week, up to 3-4 times a month; $3=2-4$ times per week; $4=$ once per day; $5=$ several times per day). The maximum total score was 15 points for each patient. Total symptom improvement was assessed by comparing the pre and post pneumatic dilation symptom scores. The concordant and discordant groups were then interviewed in person or by telephone annually for symptom recurrence. Treatment failure was defined based on patients' reports of return of their symptoms and the need for therapeutic intervention.

\section{Oesophageal manometry}

Oesophageal manometry was performed before therapy in all patients using a low compliance, pneumohydraulic, water infusion system (Arndorfer Medical Specialties, Milwaukee, Wisconsin, USA) and an eight lumen manometric catheter (Arndorfer Medical Specialties). The catheter has four proximal recording ports spaced at $5 \mathrm{~cm}$ intervals along its length and another four ports radially oriented $\left(90^{\circ}\right)$ near the tip. The recording sites were connected to an eight channel polygraph (Synectics Medical AB, Stockholm, Sweden). All swallows were monitored using an external microphone. LOS pressure was measured by the station pull through technique and recorded as the mean of four measurements at mid respiration. Completeness of LOS relaxation (normal $>85 \%$ ) was assessed as per cent decrease from mean resting LOS pressure to gastric baseline after wet swallows. Oesophageal peristalsis was recorded 3, 8, 13, and $18 \mathrm{~cm}$ above LOS in response to $5 \mathrm{ml}$ swallows of water at 30 second intervals. LOS pressures and peristalsis were measured only at the time of diagnosis.

\section{Pneumatic dilation}

All pneumatic dilations were performed using the Rigiflex balloon dilators under fluoroscopic control in the supine position. The first pneumatic dilation was usually performed with the $3.0 \mathrm{~cm}$ balloon, with progressive increases to $3.5 \mathrm{~cm}$ and $4.0 \mathrm{~cm}$ if symptoms were not relieved or if the patient had prior dilations with balloons of smaller sizes. Patients fasted for at least eight hours before the procedure. All patients were sedated with intravenous midazolam, meperidine, and topical anaesthesia of the pharynx. After upper gastrointestinal endoscopy, the Rigiflex dilator was passed over a guidewire and the balloon positioned across the diaphragmatic hiatus using the radiopaque markers as guides. Correct location was verified fluoroscopically by observing the waist, after balloon inflation pressures of 3-5 psi. The balloon was then inflated for 30-60 seconds at 9-15 psi until obliteration of the waist. A gastrografin swallow followed by a barium swallow was performed immediately after dilation to identify oesophageal perforation.

\section{Barium oesophagram}

A timed barium oesophagram ${ }^{8}$ was performed before and one month after therapy as an objective assessment of improvement in oesophageal emptying. During the six year follow up period, timed barium oesophagram was repeated in all patients with recurrence of symptoms. Additionally, 10 of the $17(59 \%)$ patients contacted in the concordant group, who were still asymptomatic, agreed to have a follow up timed barium oesophagram to assure continued concordance between symptoms and barium oesophagram. We were unable to study the remaining seven patients in this group because of loss to follow up $(n=5)$, patient's distance from the clinic $(\mathrm{n}=1)$, or time away from work $(\mathrm{n}=1)$.

All patients were asked to fast overnight prior to the barium oesophagram tests. While standing, patients ingested a low density barium sulphate suspension (45\% weight in volume) (E-Z-PAQUE; E-Z-EM, Westbury, New York, USA) over 30-45 seconds. They were instructed to drink the amount of barium they could tolerate without regurgitation or aspiration (usually between 100 and $250 \mathrm{ml}$ ). With the patient upright in a slightly left posterior oblique position, radiographs $(35 \times 35$ $\mathrm{cm}$ ) of the oesophagus were taken one, two, and five minutes after the last swallow of barium. The distance in centimetres from the distal oesophagus (identified by the bird's beak appearance of the oesophagogastric junction) to the top of a distinct barium column (barium height) as well as the maximal oesophageal width were measured. We used barium height at five minutes to determine completeness of emptying. This was based on our observations that most healthy individuals have barium emptied out of their oesophagus by one minute, and in all individuals by five minutes.

\section{Statistics}

The degree of improvement after treatment was determined as the percentage reduction of either symptoms or barium height compared with pretreatment values. Improvement in patient symptoms as well as oesophageal barium emptying was classified into three groups: (1) failure: $<50 \%$ improvement; (2) partial response: $51-90 \%$ improvement; or 3) success: 91-100\% improvement. Three possible outcomes for both subjective (symptom) as well as objective (barium emptying) improvement resulted in a $3 \times 3$ table with nine possible outcomes for each treated patient. Subjective and objective improvements were strongly associated if improvement in 
Table 1 Patient characteristics

\begin{tabular}{lccl}
\hline & $\begin{array}{l}\text { Group A } \\
\text { (discordant } \\
\text { group) }\end{array}$ & $\begin{array}{l}\text { Group B } \\
\text { (concordant } \\
\text { group) }\end{array}$ & p Value \\
\hline Sex (F/M) & $5 / 5$ & $10 / 12$ & 0.7 \\
Age (y) & $56.0(4.0)$ & $40.1(5.0)$ & 0.03 \\
LOS pressure (mm Hg) & $44.1(4.2)$ & $41.2(4.8)$ & 0.7 \\
Amplitude (mm Hg) & $24.8(2.9)$ & $30.0(17.8)$ & 0.6 \\
Barium height $(\mathrm{cm})$ & $13.0(2.7)$ & $16.1(2.0)$ & 0.4 \\
Barium height $(\mathrm{cm})^{*}$ & $10.0(2.3)$ & $3.1(1.1)$ & 0.001 \\
Barium width $(\mathrm{cm})$ & $5.8(0.7)$ & $5.0(0.4)$ & 0.3 \\
Barium width $(\mathrm{cm})^{*}$ & $5.4(0.6)$ & $3.8(0.3)$ & 0.04 \\
Long term follow up (y) & $18(3.6)$ & $59(4.8)$ & 0.001 \\
$\quad$ (range) & $3-72$ & $3-72$ & \\
\hline
\end{tabular}

Values are (mean (SEM)).

*Post therapy results.

LOS, lower oesophageal sphincter.

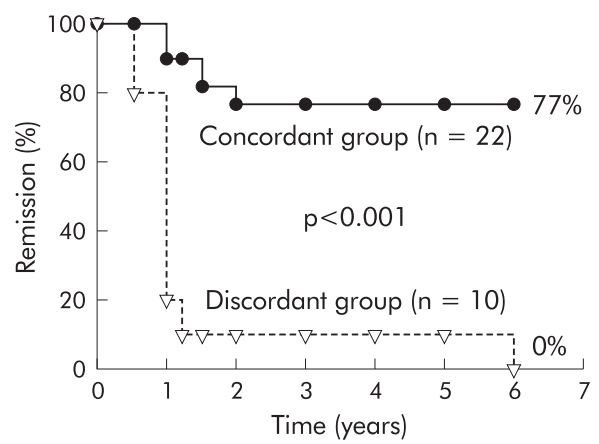

Figure 1 Kaplan-Meier graph of the remission rate (\%) in the concordant and discordant groups of achalasia patients. Over a six year follow up period, significantly $(\mathrm{p}<0.001)$ more patients in the concordant group $(17 / 22 ; 77 \%)$ were in symptom remission than in the discordant group $(0 / 10 ; 0 \%)$.

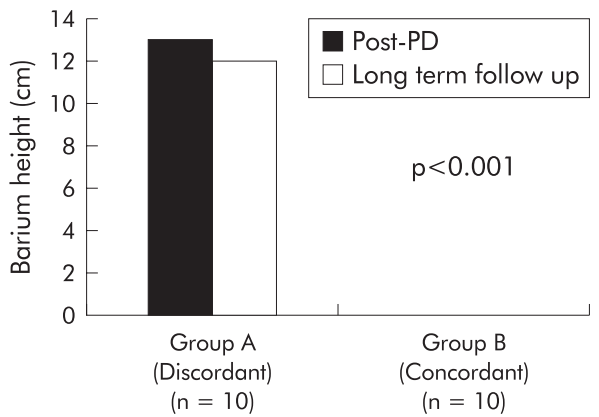

Figure 2 Oesophageal barium emptying in the discordant and concordant groups of achalasia patients at one month post pneumatic dilation (PD) compared with long term follow up values. Despite reporting near complete symptom relief post $P D$, the discordant group of patients had poor oesophageal emptying at one month post therapy, which continued long term until the report of symptom failure $(n=10)$. In the concordant group, symptom relief post PD accompanied by complete barium emptying at one month post PD persisted long term in patients available for follow up oesophagram $(n=10)$.

\section{Demographics}

Table 1 lists the demographic characteristics of the 32 patients with near complete symptom relief post therapy who were followed long term. Mean (SEM) age of the 32 patients was 46.1 (3.6) years. Mean (SEM) LOS pressure and oesophageal amplitude were 42.2 (4.4) $\mathrm{mm} \mathrm{Hg}$ and 27.2 (6.2) $\mathrm{mm} \mathrm{Hg}$, respectively. Mean (SEM) pretreatment oesophageal barium height and width were $15.1(2.2) \mathrm{cm}$ and $5.4(0.4) \mathrm{cm}$, respectively. Twenty four patients $(75 \%)$ required only one dilation while the remaining eight patients $(25 \%)$ were treated with more than one pneumatic dilation. Nineteen $(60 \%)$ of the pneumatic dilations were done using the $3.0 \mathrm{~cm}$ Rigiflex balloon, $10(31 \%)$ with the $3.5 \mathrm{~cm}$, and three (9\%) with the $4.0 \mathrm{~cm}$ balloon. There were no oesophageal perforations.

\section{Symptom to oesophageal barium improvement relationship}

Overall, in the initial 46 achalasia patients, ${ }^{8}$ there was a significant association $(\mathrm{p}<0.001$, Cochran Mantel-Haenszel) and correlation (linear regression $r=0.61, \mathrm{p}<0.001$ ) between patient symptoms and barium height improvement. However, near complete symptomatic improvement (91-100\%) was less predictive of oesophageal barium emptying. In 34 patients reporting nearly complete symptom resolution, 10 (30\%) had less than $50 \%$ oesophageal barium emptying (group A, discordant group), oesophageal emptying was moderate (50$90 \%$ ) in two patients, while 22 patients (65\%) had complete (91-100\%) improvement in both symptoms and barium height (group B; concordant group). Table 1 lists the pre and post therapy patient demographics for the discordant and concordant groups of patients. Comparing group A patients with group B patients, stepwise regression identified age as the only difference between the two groups for pre therapy parameters tested (table 1): group A patients were significantly $(p=0.03)$ older than patients in group B. Six of 10 $(60 \%)$ group A patients were older than 60 years compared with only three of 22 ( $14 \%$ ) of group B patients. By definition, group B patients had significantly $(\mathrm{p}<0.001)$ reduced oesophageal barium height and width post therapy than group A patients (table 1).

\section{Long term clinical outcome}

Mean (SEM) follow up (months) for group A (discordant) and group B (concordant) patients were 18 (3.6) (range 3-72) and 59 (4.8) (range 3-72) (table 1). Long term follow up of both groups showed that significantly $(p<0.001)$ more patients in group B $(17 / 22 ; 77 \%)$ were still in symptom remission than in group A (0/10; $0 \%$ ) (fig 1). In fact, $9 / 10(90 \%)$ group A patients failed therapy within the first 12 months of pneumatic dilation compared with $2 / 22(9 \%)$ group B patients $(p<0.001)$. Length of time in symptom remission (mean $($ SEM)) was significantly $(\mathrm{p}<0.001)$ less for group A group (18.0 (3.6) months) than for group B patients (59.0 (4.8) months) (table 1). Repeat timed barium oesophagram after report of symptom failure in group A patients $(n=10)$ on long term follow up showed continued poor oesophageal barium emptying which was similar to the one month post PD values where patients reported near complete symptom resolution (fig 2). In 10 of 17 (59\%) group B patients reporting continued 

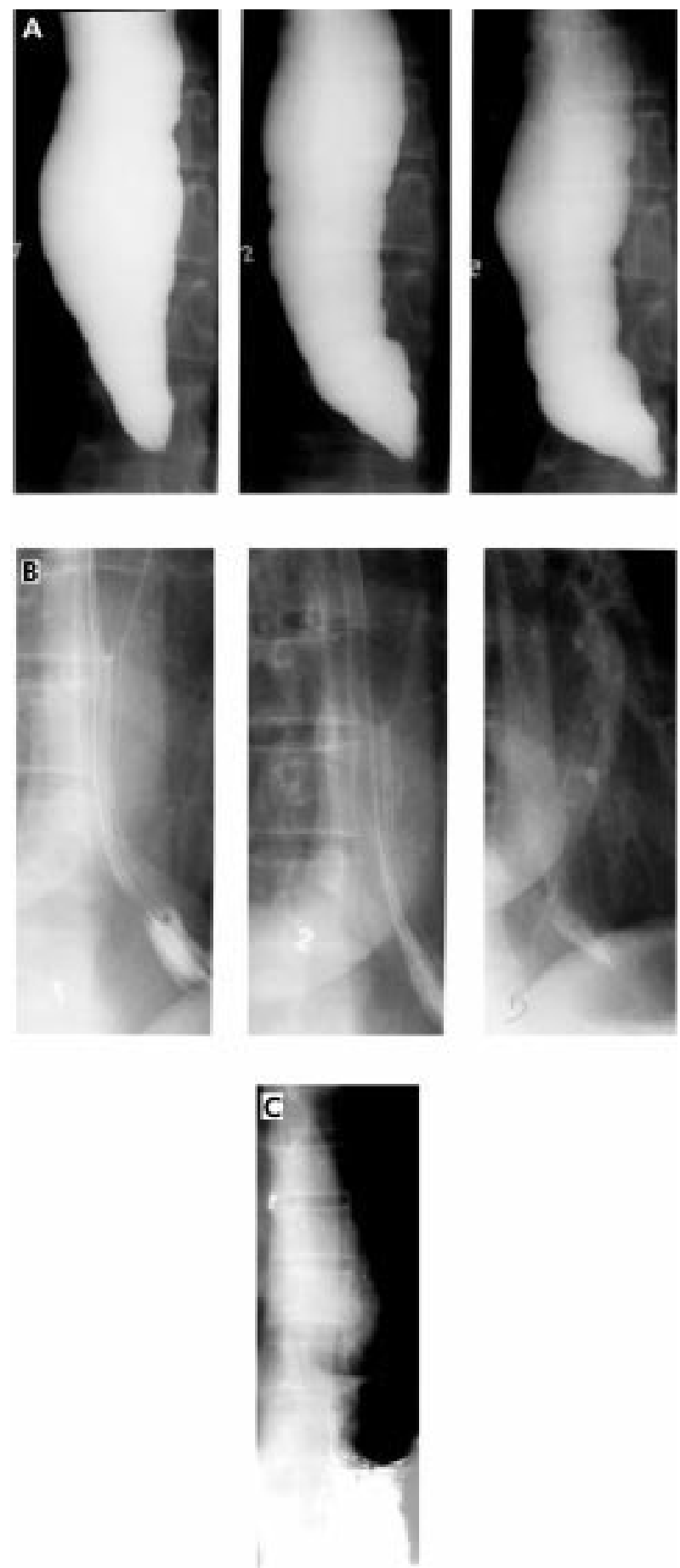

Figure 3 A 42 year old female with an initial symptom score of 11 and significant oesophageal barium retention $(A)$ underwent pneumatic dilation with the $3.0 \mathrm{~cm}$ Rigiflex balloon. She had complete symptom relief one month after treatment and her barium oesophagram showed complete oesophageal emptying by one minute (B), illustrating the concordance between symptoms and the degree of improvement in barium height. Four years after the initial pneumatic dilation the patient continues to be symptom free and timed barium oesophagram confirmed the subjective report with complete emptying by one minute $(\mathrm{C})$.

symptomatic improvement long term, timed barium oesophagram was repeated to ensure adequate objective oesophageal emptying. In all (100\%) patients, there were continued rapid oesophageal barium clearance by five minutes similar to one month post PD (fig 2). One of two patients with moderate
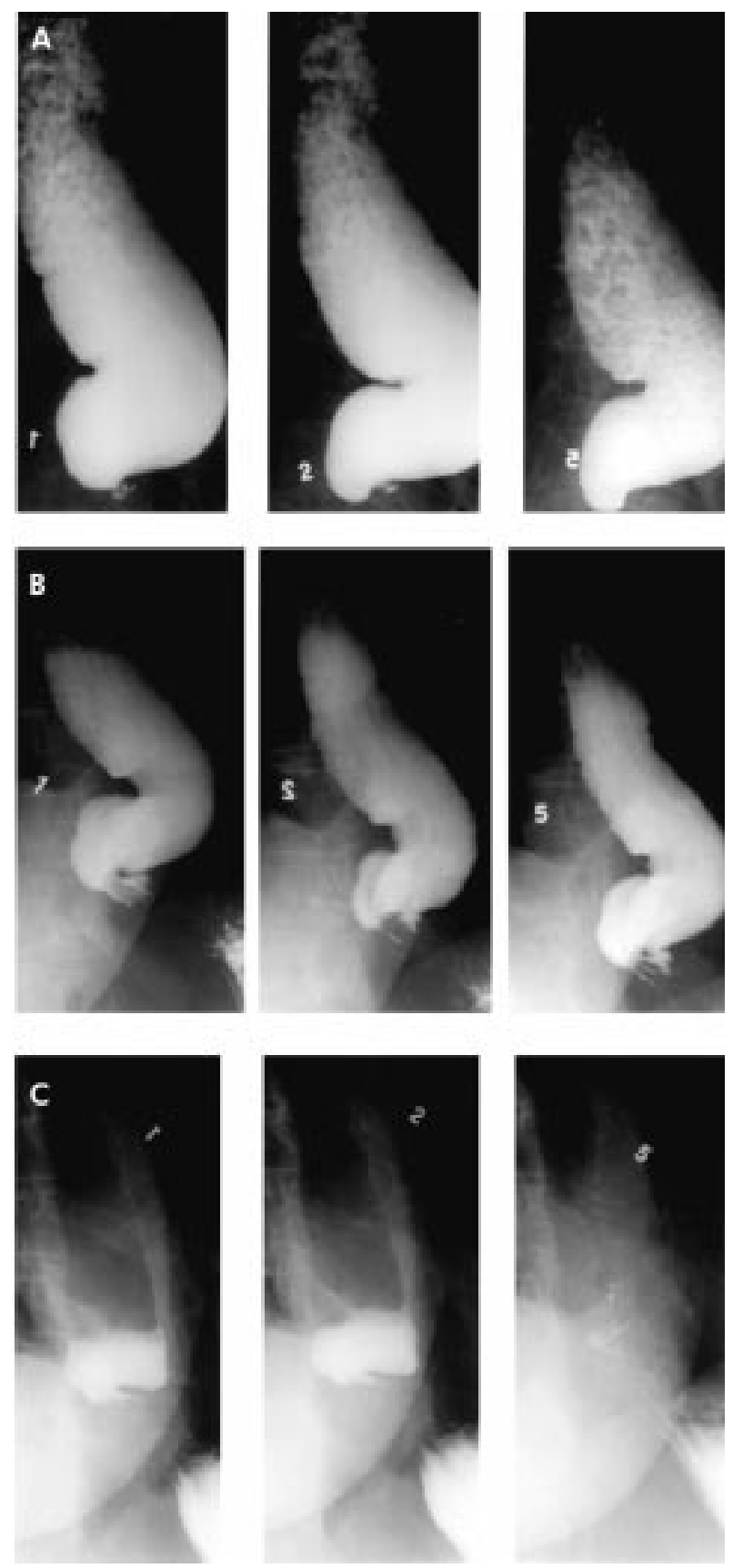

Figure 4 A 74 year old female with a pre therapy symptom score of 9 and significant oesophageal barium retention (A) underwent treatment with the $3.0 \mathrm{~cm}$ Rigiflex balloon with near complete (95\%) improvement in her symptoms. However, oesophageal barium emptying (B) showed minimal improvement compared with pre therapy levels. The patient had symptom recurrence after seven months of follow up requiring therapy. Repeat dilation with a $3.5 \mathrm{~cm}$ balloon relieved all her symptoms and oesophageal barium emptying was complete by five minutes (C), emphasising the superiority of timed barium oesophagram in assessing success post pneumatic dilation.

(50-90\%) oesophageal emptying continued to do well symptomatically at two years of follow up while the other patient was lost to follow up.

The importance of the barium oesophagram in predicting treatment success post pneumatic dilation may be demonstrated by the following two cases: (1) 42 year old female patient with an initial symptom score of 11 who had concordant symptom and timed barium oesophagram after pneumatic dilation (fig 3), and (2) 74 year old female patient with 
an initial symptom score of 9 with discordant subjective and objective findings (fig 4 ).

\section{DISCUSSION}

As we previously reported, ${ }^{9}$ the upright timed barium oesophagram, as an objective measure of oesophageal emptying, correlates well with symptom improvement observed by most achalasia patients after pneumatic dilation. In our previously reported series ${ }^{8}$ there was $73 \%$ concordance between the degree of symptom improvement and degree of oesophageal emptying by barium oesophagram. Furthermore, patients reporting poor symptom improvement $(<50 \%)$ post pneumatic dilation nearly always (17/19, 89\%) had poor oesophageal emptying on their barium examination. Somewhat to our surprise, we found that marked symptom improvement (91-100\%) after pneumatic dilation was not always accompanied by improved oesophageal emptying - that is, 10/34 (30\%) had $<50 \%$ objective improvement in their oesophageal emptying.

The finding in our previous report ${ }^{8}$ is clinically important only if those with discordant findings (group A) fail therapy earlier than those showing concordance between symptom report and oesophageal emptying (group B). The current study is the long term follow up of our patients for up to six years. We found that $77 \%$ of the concordant group were still in symptomatic remission while this was the case in none of the discordant group (fig 1). In fact, 9/10 (90\%) patients with discordant findings failed therapy within one year of pneumatic dilations (fig 1) while the failure rate was constant and slow for the concordant group. Furthermore, patients in the concordant group available for follow up continued to have complete oesophageal emptying as well as symptomatic remission long term (fig 2). Repeated barium oesophagram in 10 of these patients found that all continued to have objective improvement together with their symptomatic remission, suggesting continued and successful oesophageal decompression. Therefore, our data suggest that the timed barium oesophagram not only assesses treatment success shortly after therapy but it can also predict poor long term outcome if patients continue to have retained barium post pneumatic dilation. The latter group of patients probably warrant closer follow up than those with symptomatic and physiological improvement.

Previously reported success with Rigiflex pneumatic dilations vary from $50 \%$ to $93 \%{ }^{2}$ Until now, this variability was attributed to differences in dilation technique; however, recent reports indicate that differences in techniques (that is, balloon distention time) do not affect symptomatic outcome. ${ }^{10}$ As prior studies used symptom improvement post therapy as the primary measure of success, we speculate that a portion of these failures after pneumatic dilation represent the subset of patients with improved symptoms but continued poor oesophageal emptying. As our study indicates, symptom assessment alone may not be the optimum method of following achalasia patients post pneumatic dilation. Employing a simple objective measure of oesophageal emptying, such as the timed barium oesophagram, may identify patients who need further therapy to ensure long term remission (fig 4).

A few studies have used manometric data post therapy to identify predictors of treatment success and long term clinical response. ${ }^{11-16}$ For example, Eckardt and colleagues ${ }^{4}$ found that a post therapy LOS pressure of $<10 \mathrm{~mm} \mathrm{Hg}$ was the single most valuable factor for predicting long term clinical response $(\mathrm{p}<0.0005)$. However, studies by Kakadia and Wong, ${ }^{13} \mathrm{Kim}$ and colleagues, ${ }^{14}$ and Shahi and colleagues ${ }^{15}$ did not find any manometric predictors of symptom response. Despite the mixed results in the literature, it is generally accepted that oesophageal manometry is a useful technique not only in diagnosing achalasia but also in providing valuable information on long term clinical response after pneumatic dilation or myotomy. In our current protocol all patients had oesophageal manometry to diagnose achalasia before therapy and had timed barium oesophagram post therapy. We recently showed that there is a good correlation between post therapy reduction in LOS pressure and barium column height in achalasia patients undergoing pneumatic dilation. ${ }^{17}$ We have learned that patients are generally more accepting of the less invasive and easily performed barium oesophagram. Furthermore, as it is more widely available, patients can have the test closer to home without the need to travel to specialty centres. Some of the clinically important disadvantages of performing oesophageal manometry limiting its use both pre and post therapy include: patient discomfort, difficulty in placement of the catheter in a megaoesophagus sometimes requiring endoscopy, and the need for serial testing in the same laboratory to ensure standardisation.

The discordant group of patients in our study were older than their counterparts who had concordance of symptom change and barium oesophagram findings (table 1). This observation is consistent with earlier reports that achalasia patients with chronic symptoms often subjectively interpret minimal improvement in oesophageal emptying as dramatic improvement in swallowing. ${ }^{12}$ Additionally, our data support a recent study by Lasch and colleagues ${ }^{6}$ showing decreased pain perception to oesophageal balloon distension in older patients compared with their younger counterparts. Therefore, the clinical decision to stop therapy based on symptoms alone may leave this subset of patients with continued poor oesophageal emptying possibly leading over time to further deterioration of oesophageal function and a gradual increase in oesophageal diameter. The role of duration of patients' symptoms prior to dilation may also be an important factor but this is a difficult parameter to measure as the disease onset is usually not well established. Thus our findings underscore the need for a simple test to objectively assess the response to achalasia treatment, especially in patients reporting symptomatic improvement.

In conclusion, the timed barium oesophagram is an important objective test in evaluating the success of achalasia treatment. Using this test, we identified a subset of patients in whom oesophageal emptying was poor despite reports of excellent symptom relief after pneumatic dilation. Furthermore, nearly all patients in this group failed therapy within one year of treatment. Therefore, we believe that the timed barium oesophagram should be used to evaluate all patients pre and post pneumatic dilation to identify those who are more likely to fail therapy and require additional intervention.

\section{Authors' affiliations}

M F Vaezi, E Achkar, J E Richter, Center for Swallowing and Esophageal Disorders, Department of Gastroenterology, Cleveland Clinic Foundation, Cleveland, Ohio, USA

M E Baker, Center for Swallowing and Esophageal Disorders, Department of Radiology, Cleveland Clinic Foundation, Cleveland, Ohio, USA

\section{REFERENCES}

1 Birgisson S, Richter JE. Achalasia: what's new in diagnosis and treatment? Dig Dis 1997;15:1-27.

2 Vaezi MF, Richter JE. Current therapies for achalasia: comparison and efficacy. J Clin Gastroenterol 1998;27:21-35.

3 Spiess $\mathbf{A E}$, Kahrilas PJ. Treating achalasia: from Wahlebone to laparoscope. JAMA 1998;280:638-42.

4 Eckardt VF, Aignherr C, Bernhard G. Predictors of outcome in patients with achalasia treated by pneumatic dilation. Gastroenterology 1992;103:1732-8

5 Wehrmann $T$, Jacobi $V$, Jung $M$, et al. Pneumatic dilation in achalasia with a low-compliance balloon: results of a 5 -year prospective evaluation Gastrointest Endosc 1995;42:31-6.

6 Lasch H, Castell DO, Castell JA. Evidence for diminished visceral pain with aging: studies using graded intraesophageal balloon distension. Am J Physiol 1997;272:G1-3.

7 Gonzalez M, Mearin F, Vasconez C, et al. Oesphageal tone in patients with achalasia. Gut 1997;41:291-6. 
8 de Oliveira JM, Birgisson S, Doinoff C et al. Timed-barium swallow: a simple technique for evaluating esophageal emptying in patients with achalasia. ANR Am J Roentgenol 1997;169:473-9.

9 Vaezi MF, Baker ME, Richter JE. Assessment of esophageal emptying post-pneumatic dilation: use of the timed-barium esophagram. Am J Gastroenterol 1999:94:1802-7.

10 Khan AA, Shah WH, Alam A, et al. Pneumatic balloon dilation in achalasia: a prospective comparison of balloon distention time. Am J Gastroenterol 1998;93:1064-7.

11 Holloway RH, Krosin G, Lange RC, et al. Radionuclide esophageal emptying of a solid meal to quantitate results of therapy in achalasia. Gastroenterology 1983;84:771-6.

12 Gelfand MD, Kozarek RA. An experience with polyethylene balloon for pneumatic dilation for achalasia. Am J Gastroenterol 1989;84:924-7.
13 Kakakia SC, Wong RKH. Graded pneumatic dilation using Rigiflex achalasia dilators in patients with primary esophageal achalasia. Am J 993.88.34-8.

$14 \mathrm{Kim} \mathrm{CH}$. Cameron AJ, Hsu Jj, et al. Achalasia: prospective evaluation of relationship between lower esophageal sphincter pressure, esophageal transit, and esophageal diameter and symptoms in response to

15 Shahi HM, Aggarwal R, Misra A, et al. Relationship of manometric findings to symptomatic response after pneumatic dilation in achalasia cardia. Indian J Gastroenterol 1998;17:19-21.

16 Alonso P, Gonzalez-Conde B, Macenille R, et al. Achalasia: the usefulness of manometry for evaluation of treatment. Dig Dis $\mathrm{Sci}$ 1999:44:536-4

17 Vaezi MF, Richter JE, Wilcox CM, et al. Botulinum toxin versus pneumatic dilation in the treatment of achalasia: a randomized trial. Gut $1999 ; 44: 231-9$

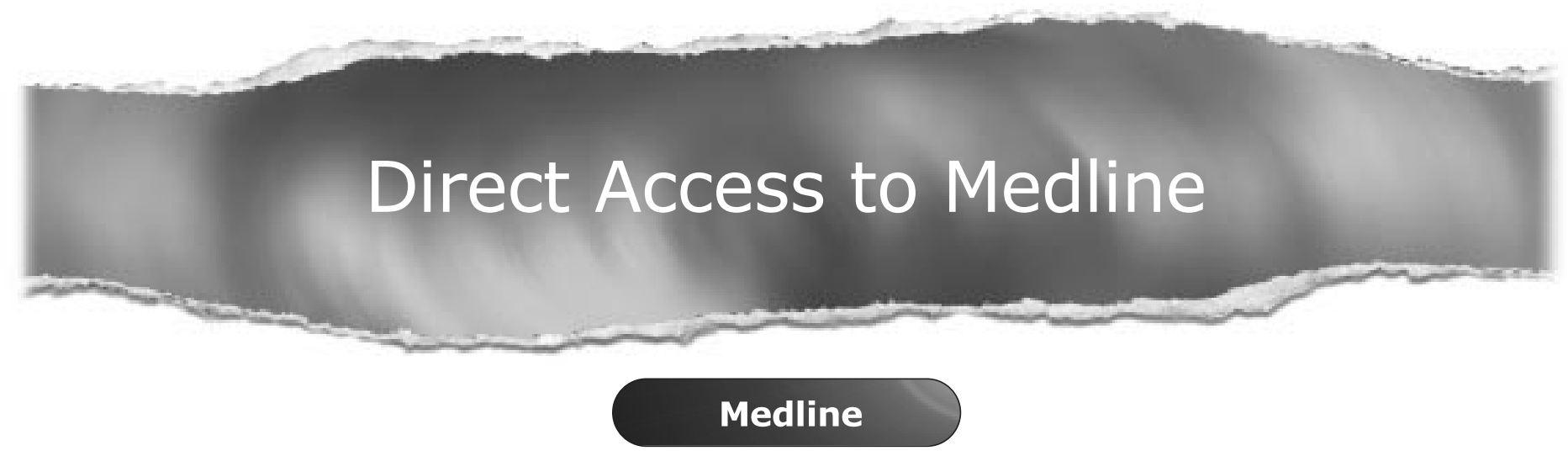

Link to Medline from the homepage and get straight into the National Library of Medicine's premier bibliographic database. Medline allows you to search across 9 million records of bibliographic citations and author abstracts from approximately 3,900 current biomedical journals.

www.gutjnl.com 